\title{
Generalized Center Problems with Outliers
}

\author{
Deeparnab Chakrabarty
}

Department of Computer Science, Dartmouth College, 9 Maynard St, Hanover, NH, USA

https://web.cs.dartmouth.edu/people/deeparnab-chakrabarty

deeparnab@dartmouth.edu

Maryam Negahbani

Department of Computer Science, Dartmouth College, 9 Maynard St, Hanover, NH, USA

maryam@cs.dartmouth.edu

\begin{abstract}
We study the $\mathcal{F}$-center problem with outliers: given a metric space $(X, d)$, a general down-closed family $\mathcal{F}$ of subsets of $X$, and a parameter $m$, we need to locate a subset $S \in \mathcal{F}$ of centers such that the maximum distance among the closest $m$ points in $X$ to $S$ is minimized.

Our main result is a dichotomy theorem. Colloquially, we prove that there is an efficient 3-approximation for the $\mathcal{F}$-center problem with outliers if and only if we can efficiently optimize a poly-bounded linear function over $\mathcal{F}$ subject to a partition constraint. One concrete upshot of our result is a polynomial time 3-approximation for the knapsack center problem with outliers for which no (true) approximation algorithm was known.
\end{abstract}

2012 ACM Subject Classification Theory of computation $\rightarrow$ Facility location and clustering

Keywords and phrases Approximation Algorithms, Clustering, k-Center Problem

Digital Object Identifier 10.4230/LIPIcs.ICALP.2018.30

Related Version A full version of the paper is available at https://arxiv.org/abs/1805. 02217.

\section{Introduction}

The $k$-center problem is a classic discrete optimization problem with numerous applications. Given a metric space $(X, d)$ and a positive integer $k$, the objective is to choose a subset $S \subseteq X$ of at most $k$ points such that $\max _{v \in X} d(v, S)$ is minimized, where $d(v, S)=\min _{u \in S} d(v, u)$. Informally, the problem is to open $k$ centers to serve all points, minimizing the maximum distance to service. This problem has been studied for at least 50 years [13, 14], is NPhard to approximate to a factor better than 2 [18], and has a simple 2-approximation algorithm $[11,16]$.

In many applications one is interested in a nuanced version of the problem where instead of serving all points in $X$, the objective is to serve at least a certain number of points. This is the so-called $k$-center with outliers version, or the robust $k$-center problem. This problem was first studied by Charikar et al. in [8] which gives a 3-approximation for the problem. A best possible 2-approximation algorithm was recently given by Chakrabarty et al. in [6] (see also the paper [15] by Harris et al. ).

Another generalization of the $k$-center problem arises when the location of centers has more restrictions. For instance, if each point in $X$ has a different weight and the constraint is that the total weight of centers opened is at most $k$. This problem, now called the knapsack center problem, was studied by Hochbaum and Shmoys in [17] which gives a 3-approximation for the problem. To take another instance, $X$ could be vectors in high dimension and the

c) (i) (1) Deeparnab Chakrabarty and Maryam Negahbani;

45th International Colloquium on Automata, Languages, and Programming (ICALP 2018). Editors: Ioannis Chatzigiannakis, Christos Kaklamanis, Dániel Marx, and Donald Sannella; Article No. 30; pp. 30:1-30:14

Leibniz International Proceedings in Informatics
LIPICS Schloss Dagstuhl - Leibniz-Zentrum für Informatik, Dagstuhl Publishing, Germany

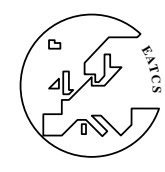
LIPICS Schloss Dagstuhl - Leibniz-Zentrum für Informatik, Dagstuhl Publishing, Germany 
centers picked need to be linearly independent vectors. This motivates the matroid center problem where the set of centers must be an independent set in a matroid. Chen et al. give a 3-approximation for this problem in [10].

Naturally, the two aforementioned generalizations can be taken together. Indeed, for the robust matroid center problem, that is, the problem of picking centers which are an independent set and only $m$ points need to be served, there is a 7-approximation algorithm in [10]. This was recently improved to a 3 -approximation in [15]. The robust knapsack center problem, however, has had no non-trivial approximation algorithm till this work. Both [10] and [15] give bi-criteria 3 -approximation algorithms which violate the knapsack constraint by $(1+\varepsilon)$ (the running time of their algorithm is exponential in $1 / \varepsilon$ ).

\section{Our Contributions}

Motivated by the state-of-affairs of the robust knapsack center problem, we study a broad generalization of the problems mentioned above. Let $\mathcal{F}$ be a general down-closed ${ }^{1}$ family of subsets over $X$. In the robust $\mathcal{F}$-center problem we are given a metric space $(X, d)$, a parameter $m$, and the objective is to select a subset $S \in \mathcal{F}$ such that $\min _{T \subseteq X,|T|=m} \max _{v \in T} d(v, S)$ is minimized. That is, the maximum distance of service of the closest $m$ points is minimized.

Observe that if $\mathcal{F}:=\{A: w(A) \leq k\}$ then we get the robust knapsack center problem, and if $\mathcal{F}$ is the collection of independent sets of a matroid, then we get the robust matroid center problem. But this generalization captures a host of other problems. For instance, one can consider multiple (but constant) knapsack constraints. Indeed, this was studied in both [17] and [10]. The former ${ }^{2}$ only looks at the version without outliers and gives a polynomial time 3-approximation in the case when the weights are all polynomially bounded. The latter proves that when the weights are not polynomially bounded, there can be no approximation algorithm via a reduction to the SUBSET SUM problem, and gives a 3-approximation violating each knapsack constraint by at most $(1+\varepsilon)$ multiplicative factor.

Another instance is a single knapsack constraint along with a single matroid constraint. To our knowledge, this problem has not been studied earlier even in the case when outliers are not allowed. This problem seems natural: for instance, when the points are high dimensional vectors with weights and the collection of centers needs to be a linearly independent set with total weight at most $k$.

The complexity of the robust $\mathcal{F}$-center problem naturally depends on the complexity of $\mathcal{F}$. To understand this, we define the following optimization problem which depends only on the set-system $(X, \mathcal{F})$. We call it the $\mathcal{F}$-maximization under partition constraints or simply $\mathcal{F}$-PCM. In this problem, one is given an arbitrary partition $\mathcal{P}$ of $X$ along with $\mathcal{F}$, and a poly-bounded (the range is at most a polynomial in $|X|$ ) value val $(x)$ on each $x \in X$. The objective is to find a set $S \in \mathcal{F}$ maximizing $\operatorname{val}(S)$ such that $S$ contains at most one element from each part of $\mathcal{P}$. Our main result stated colloquially (and formally stated as Theorem 4 and Theorem 5 in Section 2) is the following dichotomy theorem ${ }^{3}$.

1 if $A \in \mathcal{F}$ and $B \subseteq A$, then $B \in \mathcal{F}$.

2 The complete proofs can be found in the STOC 1984 version of [17]

${ }^{3}$ We are deliberately being inaccurate here. We should state the theorem for the more general supplier version where the set $X$ is partitioned into $F \cup C$ and only the points in $C$ need to be covered and only the centers in $F$ can be opened. Being more general, the algorithmic results are therefore stronger. On the other hand, we weren't able (and didn't try too hard) to make our hardness go through for the center version. In the Introduction we stick with the center version and switch to the supplier in the more formal subsequent sections. 
- Informal Theorem. For any down-closed family $(X, \mathcal{F})$, the robust $\mathcal{F}$-center problem has an efficient 3 -approximation algorithm if the $\mathcal{F}$-PCM problem can be solved in polynomial time. Otherwise, there is no efficient non-trivial approximation algorithm for the robust $\mathcal{F}$-center problem.

Note that in general, we are not concerned about how $\mathcal{F}$ is represented, because the only place the algorithm checks if a set $S$ is in $\mathcal{F}$ is perhaps for solving the $\mathcal{F}$-PCM problem. So one can choose a representation that works best for the $\mathcal{F}$-PCM solver.

A series of corollaries follow from the above theorem. These are summarized in Table 1.

- When $\mathcal{F}=\{A: w(A) \leq k\}$, the $\mathcal{F}$-PCM problem can be solved in polynomial time via dynamic programming. This crucially uses that the val is poly-bounded. Therefore we get a 3-approximation for the robust knapsack center problem. (Theorem 17)

- When $\mathcal{F}$ is the independent set of a matroid, then the $\mathcal{F}$-PCM problem is a matroid intersection problem. Therefore we get a 3-approximation for the robust matroid center problem recovering the result from [15]. (Theorem 19)

- When $\mathcal{F}=\left\{A: w_{1}(A) \leq k_{1}, w_{2}(A) \leq k_{2}, \ldots, w_{d}(A) \leq k_{d}\right\}$ is defined by $d$ weight functions and each weight function $w_{i}$ is poly-bounded, then $\mathcal{F}$-PCM can be solved efficiently using dynamic programming. Therefore we get a 3-approximation algorithm for the robust multi-knapsack center problem, extending the result in [17] to the case with outliers. (Theorem 18)

- When $\mathcal{F}$ is given by the intersection of a single knapsack and a single matroid constraint, then we don't know the complexity. However, when the weight function $w(\cdot)$ is polybounded and the matroid is representable, then we can give a randomized algorithm for the $\mathcal{F}$-PCM problem via a reduction to the exact matroid intersection problem. Therefore, we get a randomized 3-approximation for this special case of robust knapsack-and-matroid center problem (Theorem 21).

Remark 1: The Zero Outlier Case. At this juncture, the reader may wonder about the complexity of the $\mathcal{F}$-center problem which doesn't allow any outliers. This is related to the following decision problem. Given $(X, \mathcal{F})$ and an arbitrary sub-partition $\mathcal{P}$ of $X$, the problem asks whether there is a set $S \in \mathcal{F}$ such that $S$ contains exactly one element from each part of $\mathcal{P}$. We call this the $\mathcal{F}$-feasibility under partition constraints or simply the $\mathcal{F}$-PCF problem. Analogous to the informal theorem from earlier, the $\mathcal{F}$-center problem (without outliers) has an efficient 3 -approximation algorithm if the $\mathcal{F}$-PCF problem can be solved efficiently; otherwise the $\mathcal{F}$-center problem has no non-trivial approximation algorithm. Indeed, this theorem is much simpler to prove and arguably the roots of this lie in [17].

This raises the main open question from our paper: what is the relation between the $\mathcal{F}-P C F$ and the $\mathcal{F}-P C M$ problem? Clearly, the $\mathcal{F}$-PCF problem is as easy as the $\mathcal{F}$-PCM problem (set all values equal to one in the latter). But is there an $\mathcal{F}$ such that $\mathcal{F}$-PCM is "hard" while $\mathcal{F}-\mathrm{PCF}$ is "easy"? One concrete example is the corollary discussed in the last bullet point above. When $\mathcal{F}$ is a single knapsack constraint and a single matroid constraint, then the $\mathcal{F}$-PCF problem is solvable in polynomial time by minimizing a linear function over a matroid polytope and another partition matroid base polytope. As noted above, we don't know the complexity of the $\mathcal{F}$-PCM problem in this case.

Remark 2: Handling Approximations. If the $\mathcal{F}-\mathrm{PCM}$ problem is NP-hard, then the robust $\mathcal{F}$-center has no non-trivial approximation algorithm. However approximation algorithms for $\mathcal{F}$-PCM translate to bi-criteria approximation algorithms for the robust $\mathcal{F}$-center problem. More precisely, if we have a $\rho$-approximation for the $\mathcal{F}$-PCM problem $(\rho \leq 1)$, then we get 
Table 1 All the above results can be obtained as corollary or simple extensions to our main result. The numbers in bold indicate new results.

\begin{tabular}{|c|c|c|}
\hline The constraint system $\mathcal{F}$ & Without Outliers & Robust (With Outliers) \\
\hline Knapsack Constraint & $3[17]$ & $\mathbf{3}$ (Theorem 17) \\
\hline Matroid Constraint & $3[10]$ & $3[15]$ \\
\hline $\begin{array}{c}\text { Multiple Knapsack } \\
\text { poly-bounded weights) }\end{array}$ & $3[17]$ & $\mathbf{3}$ (Theorem 18) \\
\hline Knapsack and Matroid & $\mathbf{3}$ (Theorem 24) & $\begin{array}{c}\text { Open } \\
\text { special case (Theorem 21) }\end{array}$ \\
\hline $\begin{array}{c}\text { Multiple Knapsacks } \\
\text { and Matroid Constraint }\end{array}$ & $\begin{array}{c}\text { No uni-criteria } \\
\text { approximation }\end{array}$ & $\mathbf{3},(1+\varepsilon)$ violating (Theorem 27) \\
\hline
\end{tabular}

a $(3, \rho)$-bi-criteria approximation algorithm for the robust $\mathcal{F}$-center problem. That is, we return a solution $S \in \mathcal{F}$ such that the maximum distance among the closest $\rho \cdot m$ points is at most 3 times the optimum value.

There could be a different notion of approximation possible for the $\mathcal{F}$-PCM problem. Given an instance, there may be an algorithm which returns a set $S$ whose value is at least the optimum value but $S \in \mathcal{F}^{R}$ for some $\mathcal{F}^{R} \supseteq \mathcal{F}$ which is a 'relaxation' of $\mathcal{F}$. For instance, if $\mathcal{F}$ is the intersection of multiple (constant) knapsack constraints which are not poly-bounded, then for any constant $\varepsilon>0$ the $\mathcal{F}$-PCM problem can be solved $[9,12]$ returning a set with value at least the optimum but violating each constraint by multiplicative $(1+\varepsilon)$. We can use the same to get a polynomial time 3-approximation for the robust multiple knapsack-center problem if we are allowed to violate the knapsack constraints by $(1+\varepsilon)$.

\section{Our Technique}

Although our theorem statement is quite general, the proof is quite easy. Let us begin with the $\mathcal{F}$-center problem without outliers. For this, we follow the algorithmic 'partitioning' idea outlined in paper [17] by Hochbaum and Shmoys. As is standard, we guess the optimum distance which we assume to be 1 by scaling. Initially, all points are marked uncovered. Subsequently, we pick any uncovered point $x$ and consider a subset $B_{x}$ of points within distance 1 from it. Note that the optimum solution must pick at least one point from each $B_{x}$ to serve $x$. Next, we call $x$ "responsible" for all uncovered points within a distance 2 from it, and mark all these points covered. Observe that all the newly covered points are within distance 3 from any point in $B_{x}$. We continue the above procedure till all points are marked covered. Also observe that the $B_{x}$ 's form a sub-partition $\mathcal{P}$ of the universe where each part has a responsible point. By the above two observations, we see that the $\mathcal{F}$-PCF problem must have a feasible solution with respect to $\mathcal{P}$, and any solution to the $\mathcal{F}$-PCF problem gives a 3 -approximation to the $\mathcal{F}$-center problem.

Handling outliers is a bit trickier. The above argument doesn't work since the 'responsible' point may be an outlier in the optimal solution and we can no longer assert that the optimal solution must contain a point from each part. Indeed, the nub of the problem seems to be figuring out which points should be outliers. The 3-approximation algorithm in [8] by Charikar et al. (see also paper [1]) cleverly chooses the partitioning via a greedy procedure, but their argument seems hard to generalize to other constraints.

A different attack used in the algorithm in [6] by Chakrabarty et al. and that in [15] by Harris et al. is by writing an LP relaxation and using the solution of the LP to recognize 
the outliers. At a high level, the LP assigns each point $x$ a variable (in this paper we call it $\operatorname{cov}(x))$ that indicates the extent to which $x$ is served. Subsequently, the partitioning procedure described in the first paragraph is run, except the responsible points are considered in decreasing order of $\operatorname{cov}(x)$. The hope is that points assigned higher $\operatorname{cov}(x)$ in the LP solution are less likely to be outliers, and therefore the partition returned by the procedure can be used to recover a 3-approximate solution. This idea does work for the natural LP relaxation of the robust matroid center problem but fails for the natural LP relaxation of the robust knapsack center problem. Indeed, the latter has unbounded integrality gap.

Our solution is to use the round-or-cut framework that has recently been a powerful tool in designing many approximation algorithms (see $[7,20,19,2,5])$. We consider the following "coverage polytope" for the robust $\mathcal{F}$-center problem: the variables are $\operatorname{cov}(x)$ denoting the extent to which $x$ is covered by a convex combination of sets $S \in \mathcal{F}$. Of course, we cannot hope to efficiently check whether a particular cov lies in this polytope. Nevertheless, we show that for any cov in the coverage polytope, the partitioning procedure when run in the decreasing order of cov, has the property that there exists a solution $S \in \mathcal{F}$ intersecting each part at most once which covers at least $m$ points. We can then use the algorithm for $\mathcal{F}$-PCM to find this set. Furthermore, and more crucially, if the partitioning procedure does not have this property, then we can efficiently find a hyperplane separating cov from the the coverage polytope. Therefore, we can run the ellipsoid algorithm on the coverage polytope each time either obtaining a separating hyperplane, or obtaining a cov that leads to a desired partition, and therefore a 3-approximation.

\section{Preliminaries}

In this section we give formal definitions and statements of our results. As mentioned in a footnote in the Introduction, we focus on the supplier version of the problem.

- Definition 1 (F-Supplier Problem). The input is a metric space $(X, d)$ on a set of points $X=F \cup C$ with distance function $d: X \times X \longrightarrow \mathbb{R}_{\geq 0}$ and $\mathcal{F} \subseteq 2^{F}$ a down-closed family of subsets of $F$. The objective is to find $S \in \mathcal{F}$ such that $\max _{v \in C} d(v, S)$ is minimized.

- Definition 2 (Robust $\mathcal{F}$-Supplier Problem). The input is an instance of the $\mathcal{F}_{\text {-supplier }}$ problem along with an integer parameter $m \in\{0,1, \ldots,|C|\}$. The objective is to find $S \in \mathcal{F}$ and $T \subseteq C$ for which $|T| \geq m$, and $\max _{u \in T} d(u, S)$ is minimized.

Thus an instance $\mathcal{J}$ of the robust $\mathcal{F}$-supplier problem is defined by the tuple $(F, C, d, m, \mathcal{F})$. In the definitions above, $F$ and $C$ are often called the set of facilities and customers respectively.

Given the set system $\mathcal{F}$ defined over $F$, we define the following optimization problem.

- Definition 3 (F-PCM problem). The input is $\mathcal{J}=(F, \mathcal{F}, \mathcal{P}$, val) where $F$ is a finite set and $\mathcal{F} \subseteq 2^{F}$ is a down-closed family, $\mathcal{P} \subseteq 2^{F}$ is a sub-partition of $F$, and val $: F \longrightarrow\{0,1,2, \cdots\}$ is an integer-valued function with maximum range $\mid$ val $\mid$ satisfying: $\forall f_{1}, f_{2} \in A \in \mathcal{P}$, val $\left(f_{1}\right)=$ $\operatorname{val}\left(f_{2}\right)$. The objective is to find:

$$
\operatorname{opt}(\mathcal{J})=\max _{S \in \mathcal{F}} \operatorname{val}(S): \quad|S \cap A| \leq 1, \quad \forall A \in \mathcal{P}
$$

The next theorem is the main result of the paper.

Theorem 4. Given a Robust $\mathcal{F}$-Supplier instance $\mathcal{J}=(F, C, d, m, \mathcal{F})$, Let $\mathcal{A}$ be an algorithm that solves any $\mathcal{F}$-PCM instance $\mathcal{J}=(F, \mathcal{F}, \mathcal{P}$, val $)$, with $\mid$ val $|\leq| C \mid$, in time bounded by $T_{\mathcal{A}}(\mathcal{J})$. Then, there is a 3-approximation algorithm for the Robust $\mathcal{F}$-Supplier instance that runs in time $\operatorname{poly}(|\mathcal{J}|) T_{\mathcal{A}}(\mathcal{J})$. 
The next theorem is the (easier) second part of the dichotomy theorem. We show that if $\mathcal{F}$-PCM cannot be solved, then the corresponding Robust $\mathcal{F}$-Supplier cannot be approximated.

- Theorem 5. Given any non-trivial approximation algorithm $\mathcal{B}$ for the Robust $\mathcal{F}$-Supplier problem that runs in time $T_{\mathcal{B}}(|\mathcal{J}|)$ on instance $\mathcal{J}$, any $\mathcal{F}$-PCM instance $\mathcal{J}=(F, \mathcal{F}, \mathcal{P}$, val) can be solved in time poly $(|\mathcal{J}|) T_{\mathcal{B}}(|\mathcal{J}|)$, where $|\mathcal{J}|=\operatorname{poly}(|\mathcal{J}|)$.

The proof can be found in the full version of the paper.

We end this section by setting a few notations used in the remainder of the paper. For any $u \in F \cup C$ we let $B_{C}(u, r)$ be the customers in a ball of radius $r$ around $u$ i.e. $B_{C}(u, r)=\{v \in C: d(u, v) \leq r\}$. Similarly, define $B_{F}(u, r)$ as the facilities in a ball of radius $r$ around $u$ i.e. for $u \in F \cup C, B_{F}(u, r)=\{f \in F: d(u, f) \leq r\}$.

\section{Algorithm and Analysis : Proof of Theorem 4}

We fix $\mathcal{J}=(F, C, d, \mathcal{F}, m)$ the instance of the Robust $\mathcal{F}$-Supplier problem. We use $\widehat{o p t}$ to denote our guess of the value of the optimal solution. Without loss of generality, we can always assume $\widehat{\mathrm{opt}}=1$ because if not, we could scale $d$ to meet this criteria. Our objective henceforth is to either find a set $S \in \mathcal{F}$ such that $|\{v \in C: d(v, S) \leq 1\}| \geq m$, or prove that $\operatorname{opt}(\mathcal{J})>1$.

There are two parts to our proof. The first part is a partitioning procedure which given an assignment $\operatorname{cov}(v) \in \mathbb{R}_{\geq 0}$ for every customer $v \in C$, constructs an instance $\mathcal{J}$ of $\mathcal{F}$-PCM. We call cov valuable if $\mathcal{J}$ has optimum value $\geq m$. Our procedure ensures that if cov is valuable, then we get a 3-approximate solution for J. This is described in Section 3.1. The second part contains the proof of Theorem 4. In particular we show how using the round-and-cut methodology using polynomially many calls to $\mathcal{A}$ (recall this is the algorithm for $\mathcal{F}-\mathrm{PCM}$ ) we can either prove opt $(\mathcal{J})>1$, or find a valuable cov. This is described in Section 3.2.

\subsection{Reduction to $\mathcal{F}$-PCM}

Algorithm 1 inputs an assignment $\left\{\operatorname{cov}(v) \in \mathbb{R}_{\geq 0}: v \in C\right\}$. It returns a sub-partition $\mathcal{P}$ of $F$ and assigns val : $F \rightarrow\{0,1, \cdots,|C|\}$ such that all the facilities in the same part of $\mathcal{P}$ get the same val. That is, it returns an $\mathcal{F}$-PCM instance $\mathcal{J}=(F, \mathcal{F}, \mathcal{P}$, val) with $\mid$ val $|\leq| C \mid$.

The algorithm maintains a set of uncovered customers $U \subseteq C$ initialized to $C$ (Line 1). In each iteration, it picks the customer $v \in U$ with maximum cov (Line 5) and adds it to set $\operatorname{Reps}_{\text {cov }}$ (Line 6). We add the set of facilities $B_{F}(v, 1)$ at distance 1 from $v$ to $\mathcal{P}$ (Line 7,8). For each such $v$, we eke out the subset $\operatorname{Chld}(v)=B_{C}(v, 2) \cap U$ of currently uncovered clients "represented" by $v$ (Line 9 ). For every facility $f \in B_{F}(v, 1)$ we define its value to be: $\operatorname{val}(f)=|\operatorname{Chld}(v)|$ (Line 10). At the end of the iteration, $\operatorname{Chld}(v)$ is removed from $U$ (Line 11) and the loop continues till $U$ becomes $\emptyset$. This way, the algorithm partitions $C$ into $\left\{\operatorname{Chld}(v): v \in \operatorname{Reps}_{\text {cov }}\right\}$ (see fact(6)). Claim 8 shows that $\mathcal{P}$ is a sub-partition of $F$.

- Fact 6. $\left\{\operatorname{Chld}(v): v \in \operatorname{Reps}_{\text {cov }}\right\}$ is a partition of $C$.

- Fact 7. For a $v \in \operatorname{Reps}_{\text {cov }}$ and any $u \in \operatorname{Chld}(v)$ line 6 of the algorithm implies $\operatorname{cov}(v) \geq$ $\operatorname{cov}(u)$.

- Claim 8. $\mathcal{P}$ constructed by Algorithm 1 is a sub-partition of $F$.

Proof. By Line 11 of the algorithm, for each $u, v \in \operatorname{Reps}_{\text {cov }}$ we have $d(u, v)>2$ hence $B_{F}(u, 1) \cap B_{F}(v, 1)=\emptyset$ implying $\mathcal{P}$ is a sub-partition of $F$. 


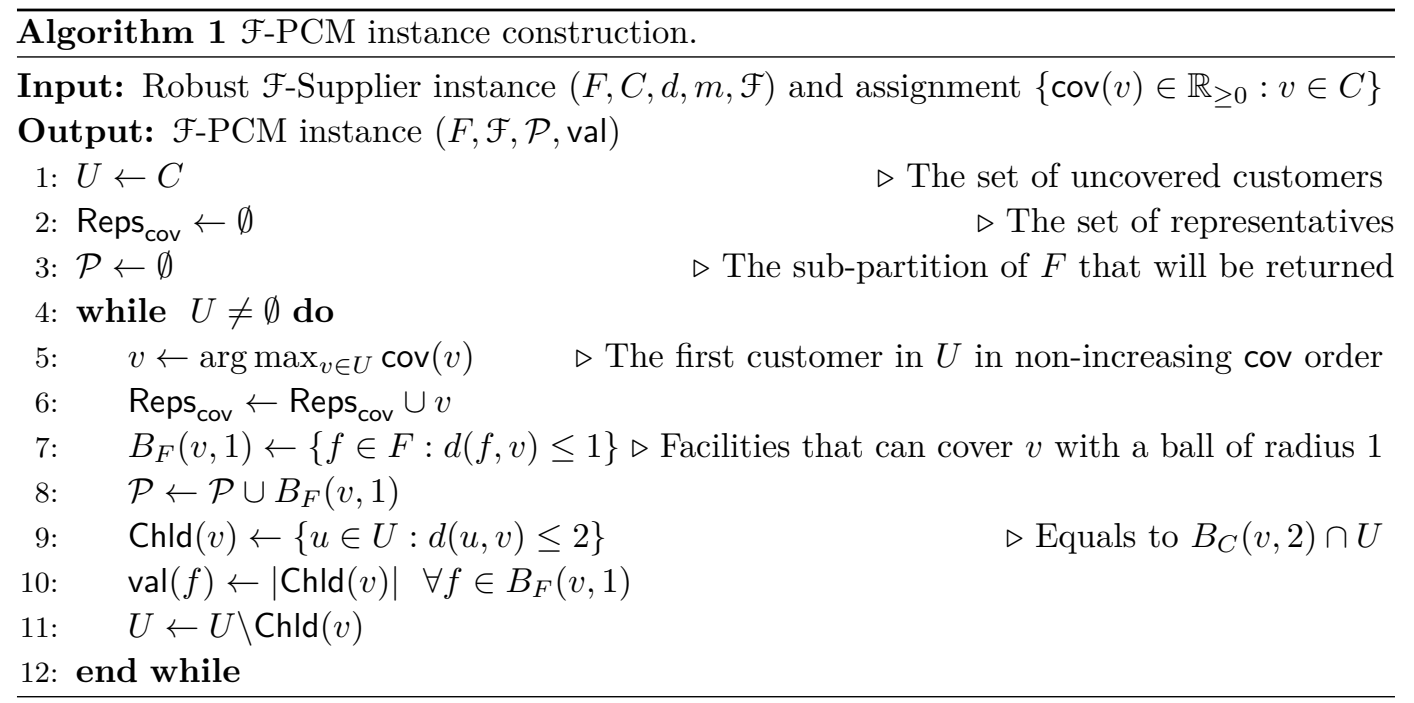

Claim 9. For each $v \in \operatorname{Reps}_{\text {cov }}$ and $f \in B_{F}(v, 1)$, Chld $(v) \subseteq B_{C}(f, 3)$.

Proof. For any $u \in \operatorname{Chld}(v)$, we have $d(u, v) \leq 2$ and since $d(f, v) \leq 1$, the fact that $d$ is metric implies $d(f, u) \leq 3$.

- Definition 10. For $S \subseteq F$ let $R(S)=\left\{v \in \operatorname{Reps}_{\text {cov }}: B_{F}(v, 1) \cap S \neq \emptyset\right\}$, be the set of representative customers in $\operatorname{Reps}_{\text {cov }}$ that are covered by balls of radius 1 around the facilities in $S$.

- Claim 11. Let $S \in \mathcal{F}$ be any feasible solution of the $\mathcal{F}$-PCM instance constructed by Algorithm 1. Then, $\sum_{f \in S} \operatorname{val}(f)=\sum_{v \in R(S)}|\operatorname{Chld}(v)|$.

Proof. For an $f \in S$, according to Line 10 of the algorithm, $\operatorname{val}(f)>0$ only if $f \in B_{F}(v, 1)$ for some $v \in \operatorname{Reps}_{\text {cov }}$. Also, by definition of the $\mathcal{F}$-PCM problem, $\left|B_{F}(v, 1) \cap S\right| \leq 1$ for any $v \in \operatorname{Reps}_{\text {cov }}$. That is, there is exactly one $f \in B_{F}(v, 1) \cap S$ for each $v \in R(S)$ and again by line $10, \operatorname{val}(f)=|\operatorname{Chld}(v)|$. Summing this equality over all $v \in R(S)$ and the corresponding $f \in B_{F}(v, 1) \cap S$ proves the claim.

- Claim 12. Let $\mathcal{J}=(F, C, d, m, \mathcal{F})$ be a Robust $\mathcal{F}$-Supplier instance and let cov $: C \rightarrow \mathbb{R}_{>0}$ be a coverage function. Let $\mathcal{J}=(F, \mathcal{F}, \mathcal{P}$, val) be the $\mathcal{F}-P C M$ instance returned by Algorithm 1 on input $\mathcal{J}$ and cov. Given any feasible solution $S$ to $\mathcal{J}$, we can cover at least val $(S)$ customers of $C$ by opening radius 3 -balls around each facility in $S$.

Proof. By considering $R(S)$ from Definition 10, Claim 11 gives: $\sum_{v \in R(S)} \mid$ Chld $(v) \mid=$ $\sum_{f \in S} \operatorname{val}(f)$. From Fact 6 , we get that for all $u, v \in \operatorname{Reps}_{\text {cov }}$, $\operatorname{Chld}(u) \cap \operatorname{Chld}(v)=\emptyset$. Thus, $\left|\bigcup_{v \in R(S)} \operatorname{Chld}(v)\right|=\sum_{v \in R(S)}|\operatorname{Chld}(v)|=\operatorname{val}(S)$. Furthermore, by Claim $9,\{v \in C: d(v, S) \leq$ $3\} \supseteq \bigcup_{u \in R(S)} \mathrm{Chld}(u)$ implying the size of the former is at least val $(S)$, thus proving the lemma.

The above claim motivates the following definition of valuable cov assignments, and the subsequent lemma.

- Definition 13. An assignment $\left\{\operatorname{cov}(v) \in \mathbb{R}_{>0}: v \in C\right\}$ is valuable with respect to a Robust $\mathcal{F}$-Supplier instance $\mathcal{J}=(F, C, d, m, \mathcal{F})$, iff opt $(\mathcal{J}) \geq m$, where $\mathcal{J}$ is the $\mathcal{F}$-PCM instance returned by Algorithm 1 from $\mathcal{J}$ and cov. 
- Lemma 14. Given an instance $\mathcal{J}$ of the Robust $\mathcal{F}$-Supplier problem with $\operatorname{opt}(\mathcal{J})=1$, and a valuable assignment cov with respect to it, we can obtain a 3-approximate solution in time poly $(|\mathcal{J}|)+T_{\mathcal{A}}(\mathcal{J})$ where $\mathcal{J}$ is the instance constructed by Algorithm 1 from $\mathcal{J}$ and cov.

Proof. Since cov is valuable, opt $(\mathcal{J}) \geq m$. We use solver $\mathcal{A}$ to return an optimal solution $S \in \mathcal{F}$ with $\operatorname{val}(S) \geq m$. Claim 12 implies that $S$ is a 3 -approximate solution to J.

\subsection{The Round and Cut Approach}

If the guess $\widehat{\mathrm{opt}}=1$ for $\mathcal{J}=(F, C, d, m, \mathcal{F})$ is at least opt(J), then the following polytope must be non-empty. To see this, if $S^{*} \in \mathcal{F}$ is the optimal solution to $\mathcal{J}$ then set $z_{S^{*}}:=1$ and $z_{S}:=0$ for $S \in \mathcal{F} \backslash S^{*}$.

$$
\begin{array}{rlr}
\sum_{\text {cov }}^{\mathcal{J}}=\{(\operatorname{cov}(v): v \in C): & \left(\mathcal{P}_{\text {cov }}^{\mathcal{J}} \cdot 1\right) \\
\forall v \in C, \operatorname{cov}(v)-\sum_{S \in \mathcal{F}: d(v, S) \leq 1} z_{S} & =0 & \left(\mathcal{P}_{\text {cov }}^{\mathcal{J}} \cdot 2\right) \\
\sum_{S \in \mathcal{F}} z_{S} & =1 & \left(\mathcal{P}_{\text {cov }}^{\mathcal{J}} \cdot 3\right) \\
z_{S} & \geq 0\} & \left(\mathcal{P}_{\text {cov }}^{\mathcal{J}} \cdot 4\right)
\end{array}
$$

Even though $\mathcal{P}_{\text {cov }}^{\mathcal{J}}$ has exponentially many auxiliary variables $\left(z_{S}\right.$ for all $\left.S \in \mathcal{F}\right)$, its dimension is still $|C|$. The following gives a family of valid inequalities for $\mathcal{P}_{\text {cov }}^{\mathcal{J}}$ via Farkas lemma.

- Lemma 15. Let $\lambda(v) \in \mathbb{R}$ for every $v \in C$ be such that

$$
\sum_{\substack{v \in C: \\ d(v, S) \leq 1}} \lambda(v) \leq m \quad \forall S \in \mathcal{F}
$$

Then any $\operatorname{cov} \in \mathcal{P}_{\text {cov }}^{\mathcal{J}}$ satisfies

$$
\sum_{v \in C} \lambda(v) \operatorname{cov}(v) \leq m
$$

Proof. Given $\operatorname{cov} \in \mathcal{P}_{\text {cov }}^{\mathcal{J}}$, there exists $\left\{z_{S}: S \in \mathcal{F}\right\}$ such that together they satisfy $\left(\mathcal{P}_{\text {cov }}^{\mathcal{J}} \cdot 1\right)$ $\left(\mathcal{P}_{\text {cov }}^{\mathcal{J}} \cdot 4\right)$.

$$
\begin{aligned}
\sum_{v \in C} \lambda(v) \operatorname{cov}(v) & ={ }_{\left(\mathcal{P}_{\text {cov }}^{\mathcal{J}} \cdot 2\right)} \quad \sum_{v \in C} \lambda(v) \sum_{\substack{S \in \mathcal{F} \\
d(v, S) \leq 1}} z_{S}=\sum_{S \in \mathcal{F}} z_{S} \sum_{\substack{v \in C: \\
d(v, S) \leq 1}} \lambda(v) \\
& \leq_{(\mathrm{V} 1),\left(\mathcal{P}_{\text {cov }}^{\mathcal{J}} \cdot 4\right)} m \sum_{S \in \mathcal{F}} z_{S}={ }_{\left(\mathcal{P}_{\text {cov }}^{\mathcal{J}} \cdot 3\right)} m
\end{aligned}
$$

The next lemma shows that all cov's in $\mathcal{P}_{\text {cov }}^{\mathcal{J}}$ are valuable.

- Lemma 16. Suppose an assignment $\left\{\operatorname{cov}(v) \in \mathbb{R}_{\geq 0}: v \in C\right\}$ is not valuable with respect to $\mathcal{J}=(F, C, d, m, \mathcal{F})$. Then there is a hyper-plane separating it from $\mathcal{P}_{\text {cov }}^{\mathcal{J}}$ that can be constructed in polynomial time. 
Proof. If $\sum_{v \in C} \operatorname{cov}(v)<m$, this inequality itself is a separating hyper-plane and we are done. So we may assume $\sum_{v \in C} \operatorname{cov}(v) \geq m$.

Let $\mathcal{J}=(F, \mathcal{F}, \mathcal{P}$, val) be the $\mathcal{F}$-PCM instance constructed by Algorithm 1 from $\mathcal{J}$ and cov. Fix $S \in \mathcal{F}$ and recall from Definition 10 that $R(S)=\left\{v \in \operatorname{Reps}_{\text {cov }}: B_{F}(v, 1) \cap S \neq \emptyset\right\}$. Pick an arbitrary $T \subseteq S$ for which $\left|B_{F}(v, 1) \cap T\right|=1$, for all $v \in R(S)$. Observe that by downclosedness of $\mathcal{F}$, we have $T \in \mathcal{F}$ which implies $T$ is a feasible solution for $\mathcal{J}$, and since cov is not valuable $\operatorname{val}(T)<m$. Furthermore, Claim 11 applied to $T$ gives val $(T)=\sum_{v \in R(T)} \mid$ Chld $(v) \mid$. Since $R(S)=R(T)$ and $|\mathrm{Chld}(v)|$ is integer-valued, we get:

$$
\sum_{v \in R(S)}|\operatorname{Chld}(v)| \leq m-1
$$

Let $\alpha=\frac{m}{m-0.5}>1$. Define $\lambda(v)$ for $v \in C$ as:

$$
\lambda(v)= \begin{cases}\alpha|\operatorname{Chld}(v)| & v \in \operatorname{Reps}_{\text {cov }} \\ 0 & \text { for all other } v \in C\end{cases}
$$

Now observe that for any $S \in \mathcal{F}$ :

$$
\sum_{v \in C: d(v, S) \leq 1} \lambda(v)=\sum_{v \in \operatorname{Reps}_{\mathrm{cov}}: d(v, S) \leq 1} \alpha|\operatorname{Chld}(v)|=\alpha \sum_{v \in R(S)}|\operatorname{Chld}(v)| \leq \alpha(m-1)<m
$$

That is, $\lambda(v)$ 's satisfy (V1). Now we prove (V2) is not satisfied thus it can be used to separate cov from $\mathcal{P}_{\text {cov }}^{\mathcal{J}}$.

$$
\begin{aligned}
& \sum_{v \in C} \lambda(v) \operatorname{cov}(v)= \\
& \alpha \sum_{v \in \operatorname{Reps}_{\mathrm{cov}}}|\operatorname{Chld}(v)| \operatorname{cov}(v)= \\
& \alpha \sum_{v \in \operatorname{Reps}_{\text {cov }}} \sum_{u \in \operatorname{Chld}(v)} \operatorname{cov}(v) \\
& \geq_{\text {Fact } 7} \alpha \sum_{v \in \operatorname{Reps}_{\text {cov }}} \sum_{u \in \operatorname{Chld}(v)} \operatorname{cov}(u)={ }_{\text {Fact } 6} \\
& \alpha \sum_{v \in C} \operatorname{cov}(v) \geq \alpha m>m
\end{aligned}
$$

Proof of Theorem 4. Given the guess opt which is scaled to 1, we use the ellipsoid algorithm to check if $\mathcal{P}_{\text {cov }}^{\mathcal{J}}$ is empty or not. Whenever ellipsoid asks if a given cov is in $\mathcal{P}_{\text {cov }}^{\mathcal{J}}$ or not, run Algorithm 1 for this given cov to construct the corresponding $\mathcal{F}$-PCM instance $\mathcal{J}$ and use algorithm $\mathcal{A}$, promised in the statement of Theorem 4 , to solve it. If opt $(\mathcal{J}) \geq m$, then Lemma 14 implies that we have a 3-approximate solution. Otherwise, cov is not valuable, and we can use Lemma 16 to find a separating hyperplane. In polynomial time, either we get a $\operatorname{cov} \in \mathcal{P}_{\text {cov }}^{\mathcal{J}}$ which by Lemma 16 has to be valuable, or we prove $\mathcal{P}_{\text {cov }}^{\mathcal{J}}$ is empty and we modify our opt guess. For the correct guess, the latter case won't occur and we get a 3-approximate solution.

\section{$4 \quad$ Applications and Extensions}

In this section we elaborate on the applications and extensions stated in the Introduction. We begin with looking at specific instances of $\mathcal{F}$ which have been studied in the literature, and some which have not.

Single and Multiple Knapsack Constraints. We look at

$$
\mathcal{F}_{\mathrm{KN}}:=\left\{S \subseteq F: \text { for } i=1, \ldots, d, \sum_{v \in S} w_{i}(v) \leq k_{i}\right\}
$$


where there are $d$ weight functions over $F$ and $k_{i}$ 's are upper bounds on these weights. Of special interest is the case $d=1$ in which we get the robust knapsack supplier problem also called the weighted $k$-supplier problem with outliers.

The $\mathcal{F}$-PCM problem for the above $\mathcal{F}_{\text {KN }}$ has the following complexity: When $d=1$, the problem can be solved in polynomial time. Indeed, given a partition $\mathcal{P}$, since $\operatorname{val}(u)=\operatorname{val}(v)$ for all $v$ in the same part, any solution which picks a facility from a part $A \in \mathcal{P}$ may as well pick the one with the smallest weight in that part. Thus, the problem boils down to the usual knapsack problem in which we have $|\mathcal{P}|$ items where the item corresponding to part $A \in \mathcal{P}$ has weight $\min _{v \in A} w(v)$ and value val $(v)$. Since the values are poly-bounded, this problem is solvable in polynomial time. Thus, we get the following corollary to Theorem 4 resolving the open question raised in [10] and [15].

- Theorem 17. There is a polynomial time 3-approximation to the robust knapsack center problem.

When $d>1$, then the $\mathcal{F}$-PCM problem is NP-hard even when val is poly-bounded. However, if the $w_{i}$ 's are also poly-bounded (actually one of them can be general), then the F-PCM problem can be solved in polynomial time using dynamic programming. This problem was in fact studied in [17] (the conference version) and is called the suitcase problem there. Thus, we get the following corollary to Theorem 4 extending the result in [17].

- Theorem 18. There is a polynomial time 3-approximation to the robust multiple-knapsack center problem if the number of weights is a constant and all but possibly one weight function are poly-bounded.

Single and Multiple Matroid Constraints. We look at

$$
\mathcal{F}_{\text {Mat }}:=\left\{S \subseteq F: S \in \mathcal{J}_{M_{i}}, \forall i=1, \ldots, d\right\}
$$

When $d=1$, we get the robust matroid center problem. The F-PCM paper reduces to finding a maximum value set in $\mathcal{J}_{M}$ and a partition matroid induced by $\mathcal{P}$. This is solvable in polynomial time even when val is general and not poly-bounded, and even when $\mathcal{J}_{M}$ is given as an independent set oracle. Thus, we get the following corollary to Theorem 4 obtaining the result in [15].

- Theorem 19. [Theorem 1.1 in [15]] There is a polynomial time 3-approximation to the robust matroid center problem even when the matroid is described as an independent set oracle.

When there are $d>1$ matroids, then the $\mathcal{F}$-PCM problem is NP-hard. Therefore, Theorem 5 implies that for instance, we can have no unicriteria approximation for the robust matroidintersection center problem.

Single Knapsack and Single Matroid Constraint. We look at

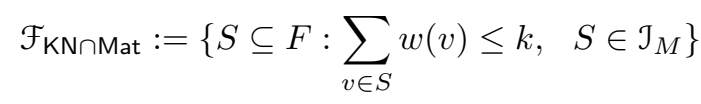

which is the intersection of a single matroid and a single knapsack constraint. To the best of our knowledge, the resulting Robust $\mathcal{F}$-Supplier problem has not been studied before. One natural instantiation is when $F$ is a collection of high-dimensional vectors with weights and the constraint on the centers is to pick a linearly independent set with total weight at most $k$. 
The corresponding $\mathcal{F}$-PCM problem asks us, given a partition $\mathcal{P}$ and poly-bounded values val, to find a set $S \in \mathcal{J}_{\mathcal{M}} \cap \mathcal{J}_{\mathcal{P}}$ of maximum value such that $w(S) \leq k$, where $\mathcal{J}_{\mathcal{P}}$ is the partition matroid induced by $\mathcal{P}$. We don't know if this problem can be solved in polynomial time, even in the case when $M$ is another partition matroid.

However, the above problem is related to the exact matroid intersection problem. In this problem, we are given two matroids $\mathcal{M}$ and $\mathcal{P}$, and a weight function $\mathbf{w}$ on each ground element and a budget $\mathbf{W}$. The objective is to decide whether or not there is a set $S \in \mathcal{J}_{\mathcal{M}} \cap \mathcal{J}_{P}$ such that $\mathbf{w}(S)=\mathbf{W}$. Understanding the complexity of this problem is a long standing challenge $[4,21,22]$. When the matroids are representable over the same field, then [4] gives a randomized pseudopolynomial time algorithm for the problem. The following claim shows the relation between $\mathcal{F}$-PCM and the exact matroid intersection problem; this claim is essentially present in [3] and the reader can refer to the full version of our paper for the proof.

- Claim 20. Given an algorithm for the exact matroid intersection problem, one can solve the $\mathcal{F}-P C M$ problem in polynomial time when the weights $w$ are poly-bounded.

Armed with the non-trivial result about exact matroid intersection from [4], we get the following.

- Theorem 21. Given a linear matroid $\mathcal{M}$ and a poly-bounded weight function, there is a randomized polynomial time 3-approximation to the robust knapsack-and-matroid center problem.

\subsection{The Case of No Outliers}

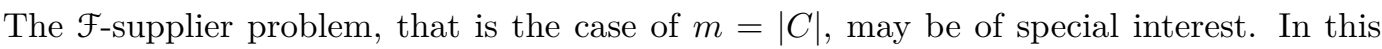
case the problem is easier and the complexity is defined by the complexity of the following decision problem.

- Definition 22 ( $\mathcal{F}$-PCF problem). The input is $\mathcal{J}=(F, \mathcal{F}, \mathcal{P})$ where $F$ is a finite set, $\mathcal{F} \subseteq 2^{F}$ is a down-closed family and $\mathcal{P} \subseteq 2^{F}$ is an arbitrary sub-partition of $F$. The objective is to decide whether there exists a set $S \in \mathcal{F}$ such that $|S \cap A|=1, \forall A \in \mathcal{P}$.

- Theorem 23. If the $\mathcal{F}-P C F$ problem can be solved efficiently for any partition $\mathcal{P}$, then the $\mathcal{F}$-supplier problem has a polynomial time 3-approximation. Otherwise, there is no non-trivial approximation possible for the $\mathcal{F}$-supplier problem.

Sketch. Run Algorithm 1 with an arbitrary assignment cov (and ignore the val's). Let $\mathcal{J}=(F, \mathcal{F}, \mathcal{P})$ be the resulting $\mathcal{F}$-PCF instance. If the guess $\widehat{\mathrm{opt}}=1$ is correct, then note that the optimum solution $S^{*}$ must satisfy $S^{*} \cap A \neq \emptyset$ for all $A \in \mathcal{P}$; if not, then the corresponding $v \in \operatorname{Reps}_{\text {cov }}$ can't be served. Conversely, any $S$ satisfying $S \cap A \neq \emptyset$ for all $A \in \mathcal{P}$ implies a 3 -approximate solution. Therefore, an algorithm for $\mathcal{F}$-PCF can either give a 3-approximate solution or prove the guess $\widehat{\text { opt }}$ is too low.

Theorem 4 and Theorem 23 raise the question: is there any set of constraints for which the problem without outliers is significantly easier than the problem with outliers? We don't know the answer to this question, although we guess the answer is yes. For this, it suffices to design a set system for which $\mathcal{F}$-PCF is easy but $\mathcal{F}$-PCM is hard (perhaps NP-hard). To see the difference between these problems consider the $\mathcal{F}_{\mathrm{KN} \cap M}$ at family described in the previous subsection. We don't know if $\mathcal{F}$-PCM is easy or hard, but $\mathcal{F}$-PCF is easy: this amounts to minimizing $w(S)$ over $S \in \mathcal{J}_{\mathcal{M}} \cap \mathcal{B}_{\mathcal{P}}$ where $\mathcal{B}_{\mathcal{P}}$ is the base polytope induced by $\mathcal{P}$. This can be done in polynomial time, and therefore we get the following corollary. 
- Theorem 24. There is a polynomial time 3-approximation to the knapsack-and-matroid center problem.

\subsection{Handling Approximation}

The technique used to prove Theorem 4 is robust enough to translate approximation algorithms for the $\mathcal{F}$-PCM problem to bi-criteria approximation algorithms for the Robust $\mathcal{F}$-Supplier problem. There are two notions of approximation algorithms for the F-PCM problem and they lead to two notions of bi-criteria approximation.

The first is the standard notion: a $\rho$-approximation (for $\rho \leq 1$ ) algorithm that takes instance $\mathcal{J}$ of $\mathcal{F}$-PCM, returns a solution $S \in \mathcal{F}$ of value $\operatorname{val}(S) \geq \rho \cdot \operatorname{opt}(\mathcal{J})$. The corresponding bi-criteria approximation notion for the Robust $\mathcal{F}$-Supplier problem is the following: an $(\alpha, \beta)$-approximation algorithm for instance $\mathcal{J}$ of Robust $\mathcal{F}$-Supplier returns a solution which opens centers at $S \in \mathcal{F}$ and the distance of at least $\beta m$ customers to $S$ is $\leq \alpha \cdot \operatorname{opt}(\mathcal{J})$. The proof of Theorem 4 in fact implies the following.

- Theorem 25. Let $\mathcal{A}$ be a polynomial time $\rho$-approximate algorithm for the $\mathcal{F}$-PCM problem. Then there is a polynomial time $(3, \rho)$-bi-criteria approximation algorithm for the Robust $\mathcal{F}$-Supplier problem.

The second notion of approximation for the $\mathcal{F}$-PCM problem is one which satisfies the constraints approximately. This notion is more problem dependent and makes sense only if there is a notion of an approximate relaxation $\mathcal{F}^{R}$ for the set $\mathcal{F}$. For example, an $(1+\varepsilon)$ relaxation for $\mathcal{F}_{\mathrm{KN}}$ could be the subsets $S$ with $w_{i}(S) \leq(1+\varepsilon) \cdot k_{i}$ for all $i$. A $\rho$-violating algorithm for an instance $\mathcal{J}$ of $\mathcal{F}$-PCM would then return a set $S$ with $\operatorname{val}(S) \geq \operatorname{opt}(\mathcal{J})$ but $S \in \mathcal{F}^{R}$ which is an $\rho$-relaxation for $\mathcal{F}$. This defines a different bi-criteria approximation notion for the Robust $\mathcal{F}$-Supplier problem. An $\alpha$-approximate $\beta$-violating algorithm for the Robust $\mathcal{F}$-Supplier problem takes an instance $\mathcal{J}$ and returns a solution $S \in \mathcal{F}^{R}$ which is a $\beta$-relaxation for $\mathcal{F}$ such that at least $m$ customers in $C$ are at distance at most $\alpha \cdot \operatorname{opt}(\mathcal{J})$ to $S$.

- Theorem 26. Let $\mathcal{A}$ be a polynomial time $\rho$-violating algorithm for the $\mathcal{F}$-PCM problem. Then there is a polynomial time 3-approximate- $\rho$-violating algorithm for the Robust $\mathcal{F}$-Supplier problem.

When $\mathcal{F}$ is described by constant $d$ knapsack constraints (with general weights) and a single matroid constraint, for any constant $\varepsilon>0$ Chekuri et al. give an $(1+\varepsilon)$-approximation algorithm for the F-PCM in [9]. Without the matroid constraint, Grandoni et al. give an $(1+\varepsilon)$-violating algorithm in [12]. Together, we get the following corollary. The latter recovers a result from [10].

- Theorem 27. Fix any constant $\varepsilon>0$. There is a polynomial time $(3,(1+\varepsilon))$-bi-criteria approximation algorithm for the robust supplier problem with constant many knapsack constraints and one matroid constraint. There is a polynomial time 3 -approximate $(1+\varepsilon)$-violating algorithm for the robust supplier problem with constant many knapsack constraints.

\section{References}

1 Gagan Aggarwal, Rina Panigrahy, Tomás Feder, Dilys Thomas, Krishnaram Kenthapadi, Samir Khuller, and An Zhu. Achieving Anonymity via Clustering. ACM Trans. Algorithms, 6(3):49:1-49:19, 2010. doi:10.1145/1798596.1798602. 
2 Hyung-Chan An, Mohit Singh, and Ola Svensson. LP-based algorithms for capacitated facility location. In Proceedings of the 2014 IEEE 55th Annual Symposium on Foundations of Computer Science, FOCS '14, pages 256-265, Washington, DC, USA, 2014. IEEE Computer Society. doi:10.1109/FOCS.2014.35.

3 André Berger, Vincenzo Bonifaci, Fabrizio Grandoni, and Guido Schäfer. Budgeted Matching and Budgeted Matroid Intersection via the Gasoline Puzzle. Mathematical Programming, 128(1):355-372, 2011. doi:10.1007/s10107-009-0307-4.

4 Paolo M. Camerini, Giulia Galbiati, and Francesco Maffioli. Random Pseudo-Polynomial Algorithms for Exact Matroid Problems. J. Algorithms, 13(2):258-273, 1992. doi:10. 1016/0196-6774 (92) 90018-8.

5 Robert D. Carr, Lisa K. Fleischer, Vitus J. Leung, and Cynthia A. Phillips. Strengthening Integrality Gaps for Capacitated Network Design and Covering Problems. In Proceedings of the Eleventh Annual ACM-SIAM Symposium on Discrete Algorithms, SODA '00, pages 106-115, Philadelphia, PA, USA, 2000. Society for Industrial and Applied Mathematics. URL: http://dl . acm. org/citation . cfm?id=338219.338241.

6 Deeparnab Chakrabarty, Prachi Goyal, and Ravishankar Krishnaswamy. The Non-Uniform $k$-Center Problem. In 43rd International Colloquium on Automata, Languages, and Programming, ICALP 2016, July 11-15, 2016, Rome, Italy, pages 67:1-67:15, 2016. doi: 10.4230/LIPIcs. ICALP. 2016.67.

7 Deeparnab Chakrabarty, Ravishankar Krishnaswamy, and Amit Kumar. The Heterogeneous Capacitated k-Center Problem. In Integer Programming and Combinatorial Optimization - 19th International Conference, IPCO 2017, Waterloo, ON, Canada, June 26-28, 2017, Proceedings, pages 123-135, 2017. doi:10.1007/978-3-319-59250-3_11.

8 Moses Charikar, Samir Khuller, David M. Mount, and Giri Narasimhan. Algorithms for Facility Location Problems with Outliers. In Proceedings of the Twelfth Annual ACMSIAM Symposium on Discrete Algorithms, SODA '01, pages 642-651, Philadelphia, PA, USA, 2001. Society for Industrial and Applied Mathematics.

9 Chandra Chekuri, Jan Vondrák, and Rico Zenklusen. Multi-budgeted Matchings and Matroid Intersection via Dependent Rounding. Proceedings of the Twenty-Second Annual ACM-SIAM Symposium on Discrete Algorithms, pages 1080-1097, 2011. doi:10.1137/1. 9781611973082.82 .

10 Danny Z. Chen, Jian Li, Hongyu Liang, and Haitao Wang. Matroid and Knapsack Center Problems. In Integer Programming and Combinatorial Optimization (IPCO), pages 110122, 2013.

11 Teofilo F. Gonzalez. Clustering to Minimize the Maximum Intercluster Distance. Theoretical Computer Science, 38:293-306, 1985. doi:10.1016/0304-3975(85)90224-5.

12 Fabrizio Grandoni, R. Ravi, Mohit Singh, and Rico Zenklusen. New Approaches to Multi-Objective Optimization. Math. Program., 146(1-2):525-554, 2014. doi:10.1007/ s10107-013-0703-7.

13 Seifollah L. Hakimi. Optimum Locations of Switching Centers and the Absolute Centers and Medians of a Graph. Ops Res.INFORMS, 12:450-459, 1964. doi:10.1287/opre.12.3.450.

14 Seifollah L. Hakimi. Optimum Distribution of Switching Centers in a Communication Network and Some Related Graph Theoretic Problems. Ops Res.INFORMS, 13:462-475, 1965. doi:10.1287/opre.13.3.462.

15 David G. Harris, Thomas Pensyl, Aravind Srinivasan, and Khoa Trinh. A Lottery Model for Center-Type Problems with Outliers. In Approximation, Randomization, and Combinatorial Optimization. Algorithms and Techniques, APPROX/RANDOM 2017, August 16-18, 2017, Berkeley, CA, USA, pages 10:1-10:19, 2017. doi:10.4230/LIPIcs . APPROX-RANDOM . 2017.10 . 
16 Dorit S. Hochbaum and David B. Shmoys. A Best Possible Heuristic for the $k$-Center Problem. Math. Oper. Res., 10(2):180-184, 1985. doi:10.1287/moor.10.2.180.

17 Dorit S. Hochbaum and David B. Shmoys. A Unified Approach to Approximation Algorithms for Bottleneck Problems. J. ACM, 33(3):533-550, 1986. doi:10.1145/5925.5933.

18 Wen-Lian Hsu and George L. Nemhauser. Easy and Hard Bottleneck Location Problems. Discrete Applied Mathematics, 1(3):209-215, 1979. doi:10.1016/0166-218X (79)90044-1.

19 Shi Li. On Uniform Capacitated $k$-median Beyond the Natural LP Relaxation. In Proceedings of the Twenty-sixth Annual ACM-SIAM Symposium on Discrete Algorithms, SODA '15, pages 696-707, Philadelphia, PA, USA, 2015. Society for Industrial and Applied Mathematics.

20 Shi Li. Approximating Capacitated $k$-median with $(1+\varepsilon) k$ Open Facilities. In Proceedings of the Twenty-seventh Annual ACM-SIAM Symposium on Discrete Algorithms, SODA '16, pages 786-796, Philadelphia, PA, USA, 2016. Society for Industrial and Applied Mathematics. URL: http://dl.acm.org/citation. cfm?id=2884435.2884491.

21 Ketan Mulmuley, Umesh V. Vazirani, and Vijay V. Vazirani. Matching is As Easy As Matrix Inversion. In Proceedings of the Nineteenth Annual ACM Symposium on Theory of Computing, STOC '87, pages 345-354, New York, NY, USA, 1987. ACM. doi:10.1145/ 28395.383347.

22 Christos H. Papadimitriou and Mihalis Yannakakis. The Complexity of Restricted Spanning Tree Problems. J. ACM, 29(2):285-309, 1982. doi:10.1145/322307.322309. 